\title{
Research on Bertrand Dual-Oligopoly Dynamic Game Model
}

\author{
Fangxu Ren \& Xiongyi Li \\ College of Economic Management, Zhong Yuan Institute of Technology, Zhengzhou 450007, China \\ E-mail: renfx12@163.com
}

\begin{abstract}
In this article, we study the equilibrium solution and the dynamic change process of the Bertrand price competition model under the condition of dual-manufactures, give the model of dynamic change, prove that whatever manufactures choose about price, the equilibrium solution only exists, and prove that the manufacture's price change process must be the monotonic process, and develop relative conclusions of the Bertrand model.
\end{abstract}

Keywords: Bertrand model, Dynamic game, Equilibrium

For $i(i=1,2, \Lambda I)$, if $u_{i}\left(\sigma_{i}^{*}, \sigma_{-i}^{*}\right) \geq u_{i}\left(s_{i j}, \sigma_{-i}^{*}\right) \forall s_{i j} \in S_{i}$ comes into existence, the mixed strategy section $\sigma^{*}$ in the problem of complete information static game is called as the Nash equilibrium of the game. The introduction of Nash equilibrium offers a base to confirm the equilibrium of the oligopoly market, and on the Nash equilibrium, various manufactures will consider their competitors and suppose their competitors will do the same thing. In 1883, French economist Joseph Bertrand utilized the concept of Nash equilibrium in the Bertrand model established by him to study the price competition, afterward that model was broad used to explain and analyze phenomena and problems about price competition, and analysis and researches about dynamic price competition game were numerous. The reference 1 gives the different oligopoly optimal orientation pure strategies and their respective optimal profits under conditions of different cost advantages. The reference 2 introduces the oligopoly dynamic game problem under the situation of information asymmetry. And many references from reference 3 to reference 6 analyze different problems from different views, but the analysis has not displayed the equilibrium solution and the dynamic change process of the oligopoly competition. In the reference 7, professor Tang Xiaowo utilizes the Cournot model to study the dynamic competition problem under dual oligopoly manufactures, but this model is on the base of output competition, more dynamic competition problems about price exist in fact.

\section{The introduction of Bertrand dual-oligopoly manufacture model}

We consider the Bertrand dual-oligopoly model and suppose that two oligopoly manufactures produce same products with different brands, different qualities and different packages, manufacture $\mathrm{A}$ and manufacture $\mathrm{B}$ respectively select price $\mathrm{p}_{\mathrm{A}}$ and $\mathrm{p}_{\mathrm{B}}$, and the demand function to the manufacture $\mathrm{i}$ is $q_{i}=f\left(P_{i}, P_{j}\right)$, and $q_{i}\left(P_{i}, P_{j}\right)=a-P_{i}+b P_{j}$, where, $\mathrm{b}(|b|<1)$ reflects the reflection degree of the manufacture i's product to the manufacture j's product (here, suppose that the price demands to the competitive manufactures' products have same influencing coefficient). We make the marginal cost as the constant $\mathrm{c}(\mathrm{c}<\mathrm{a})$ and don't consider the fixed production cost. Two manufactures select their prices at the same time, so the manufacture i's profit function is $u_{i}\left(p_{i}, p_{j}\right)=q_{i}\left(p_{i}, p_{j}\right)\left[p_{i}-c\right] \quad(i=1,2)(i \neq j)$, and from the profit function we can obtain that $P_{1}^{m}=P_{2}^{m}=P^{m}=\frac{a+c}{2-b}$ is the Nash equilibrium of the game, and here two manufactures get the profit $\left[\frac{a+(b-1) c}{2-b}\right]^{2}$ (Jean Tirole, 1997).

When the manufacturer $\mathrm{A}$ in the market first enters into the market, he sets down the product price $\mathrm{p}_{\mathrm{A}}(1)$, and the manufacturer $\mathrm{B}$ makes his optimal price $p_{B}(1)=\frac{a+c+b P_{A}(1)}{2}$ according to the demand function $q_{i\left(P_{i}, P_{j}\right)}=a-P_{i}+b P_{j}$ and the condition of profit maximization. In the same way, if the manufacturer A adjusts his price to $p_{A}(2)=P_{A}(1) \pm \Delta P_{A}(1) \quad$, the manufacturer $\mathrm{B}$ will correspondingly adjust his price to $p_{B}(2)=\frac{a+c+b\left(P_{A}(1) \pm \Delta P_{A}\right)}{2}=p_{B}(1) \pm \frac{\Delta P_{A}(1)}{2} b$. The other way round, if the manufacturer $\mathrm{B}$ adjusts his price to $p_{B}(2)=p_{B}(1) \pm \Delta P_{B}(1)$, the manufacturer $A$ will also correspondingly adjust his price to

$P_{A}(2)=\frac{a+c+b\left(P_{B}(1) \pm \Delta P_{B}\right)}{2}=P_{A}(1) \pm \frac{\Delta P_{B}(1)}{2} b$.

Therefore, for one time game, according to the hypothesis of the model, we can obtain that manufacturer j's optimal 
adjustment extent is $\frac{b}{2} \Delta P_{i}$. But in fact, the game process of two oligopoly enterprises is dynamic and multistage, and we need to review the first manufacturer's equilibrium solution and dynamic change process under the condition of initial random selection.

\section{The dynamic process and equilibrium of Bertrand price competition}

Suppose that the price that the first manufacturer (manufacturer A) enters into the market is $p_{A}(1)$, and the price that the second manufacturer (manufacturer B) enters into the market is $p_{B}(1)=\frac{a+c}{2}+\frac{b}{2} p_{A}(1)$. After that, according to the price of manufacturer $\mathrm{B}$, the manufacturer $\mathrm{A}$ adjusts his optimal price to $p_{A}(2)=\frac{a+c}{2}+\frac{b}{2} p_{B}(1)$, correspondingly, the price of manufacturer $\mathrm{B}$ is adjusted to $p_{B}(2)=\frac{a+c}{2}+\frac{b}{2} p_{A}(2)$. After $\mathrm{n}$ times adjustment, the manufacturer $\mathrm{A}$ and the manufacturer B's prices are respectively adjusted to $p_{A}(n)=\frac{a+c}{2}+\frac{b}{2} p_{B}(n-1)$ and $p_{B}(n)=\frac{a+c}{2}+\frac{b}{2} p_{A}(n)$.

And we use the vector form to analyze the price competition,

$$
\begin{aligned}
& {\left[\begin{array}{l}
p_{A}(n) \\
p_{B}(n)
\end{array}\right]=\left[\begin{array}{ll}
0 & 0 \\
\frac{b}{2} & 0
\end{array}\right]\left[\begin{array}{l}
p_{A}(n) \\
p_{B}(n)
\end{array}\right]+\left[\begin{array}{ll}
0 & \frac{b}{2} \\
0 & 0
\end{array}\right]\left[\begin{array}{l}
p_{A}(n-1) \\
p_{B}(n-1)
\end{array}\right]+\left[\begin{array}{c}
\frac{a+c}{2} \\
\frac{a+c}{2}
\end{array}\right]} \\
& \text { i.e. }\left[\begin{array}{cc}
1 & 0 \\
-\frac{b}{2} & 1
\end{array}\right]\left[\begin{array}{l}
p_{A}(n) \\
p_{B}(n)
\end{array}\right]=\left[\begin{array}{ll}
0 & \frac{b}{2} \\
0 & 0
\end{array}\right]\left[\begin{array}{l}
p_{A}(n-1) \\
p_{B}(n-1)
\end{array}\right]+\left[\begin{array}{c}
\frac{a+c}{2} \\
\frac{a+c}{2}
\end{array}\right] \\
& {\left[\begin{array}{ll}
p_{A}(n) \\
p_{B}(n)
\end{array}\right]=\left[\begin{array}{cc}
1 & 0 \\
-\frac{b}{2} & 1
\end{array}\right]^{-1}\left[\begin{array}{ll}
0 & \frac{b}{2} \\
0 & 0
\end{array}\right]\left[\begin{array}{l}
p_{A}(n-1) \\
p_{B}(n-1)
\end{array}\right]+\left[\begin{array}{cc}
1 & 0 \\
-\frac{b}{2} & 1
\end{array}\right]^{-1}\left[\begin{array}{l}
\frac{a+c}{2} \\
\frac{a+c}{2}
\end{array}\right] .} \\
& =\left[\begin{array}{ll}
0 & \frac{b}{2} \\
0 & \frac{b^{2}}{4}
\end{array}\right]\left[\begin{array}{l}
p_{A}(n-1) \\
p_{B}(n-1)
\end{array}\right]+\left[\begin{array}{c}
\frac{a+c}{2} \\
\frac{b+2}{2} \cdot \frac{a+c}{2}
\end{array}\right]
\end{aligned}
$$

Suppose that $p(n)=\left[\begin{array}{l}p_{A}(n) \\ p_{B}(n)\end{array}\right], \quad D=\left[\begin{array}{ll}0 & \frac{b}{2} \\ 0 & \frac{b^{2}}{4}\end{array}\right]$ and $E=\left[\begin{array}{c}\frac{a+c}{2} \\ \frac{b+2}{2} \cdot \frac{a+c}{2}\end{array}\right]$, so,

$p(n)=D p(n-1)+E$

$p(n)=D^{n-1} p(1)+D^{n-2} E+D^{n-3} E+\Lambda+D^{2} E+D E+E$

$=D^{n-1} p(1)+\sum_{i=0}^{n-2} D^{i} E$

It is easy to prove that $D^{i}=\left[\begin{array}{ll}0 & \left(\frac{b}{2}\right)^{2 i-1} \\ 0 & \left(\frac{b}{2}\right)^{2 i}\end{array}\right]$, and $D^{n-1}=\left[\begin{array}{ll}0 & \left(\frac{b}{2}\right)^{2 n-3} \\ 0 & \left(\frac{b}{2}\right)^{2 n-2}\end{array}\right]$ 


$$
\begin{aligned}
& \sum_{i=0}^{n-2} D^{i}=I+\sum_{i=1}^{n-2} D^{i}=\left[\begin{array}{ll}
1 & 0 \\
0 & 1
\end{array}\right]+\sum_{i=1}^{n-2}\left[\begin{array}{cc}
0 & \left(\frac{b}{2}\right)^{2 i-1} \\
0 & \left(\frac{b}{2}\right)^{2 i}
\end{array}\right] \\
& {\left[\begin{array}{cc}
1 & \frac{b}{2} \cdot \frac{1-\left(\frac{b}{2}\right)^{2 n-4}}{1-\left(\frac{b}{2}\right)^{2}} \\
0 & 1+\left(\frac{b}{2}\right)^{2} \cdot \frac{1-\left(\frac{b}{2}\right)^{2 n-4}}{1-\left(\frac{b}{2}\right)^{2}}
\end{array}\right]} \\
& p(n)=D^{n-1} p(1)+\sum_{i=0}^{n-2} D^{i} E \\
& =\left[\begin{array}{ll}
0 & \left(\frac{b}{2}\right)^{2 n-3} \\
0 & \left(\frac{b}{2}\right)^{2 n-2}
\end{array}\right]\left[\begin{array}{l}
p_{A}(1) \\
p_{B}(1)
\end{array}\right]+\left[\begin{array}{cc}
1 & \frac{b}{2} \cdot \frac{1-\left(\frac{b}{2}\right)^{2 n-4}}{1-\left(\frac{b}{2}\right)^{2}} \\
0 & 1+\left(\frac{b}{2}\right)^{2} \cdot \frac{1-\left(\frac{b}{2}\right)^{2 n-4}}{1-\left(\frac{b}{2}\right)^{2}}
\end{array}\right]\left[\begin{array}{c}
\frac{a+c}{2} \\
\frac{b+2}{2} \cdot \frac{a+c}{2}
\end{array}\right] \\
& =\left[\begin{array}{l}
\left(\frac{b}{2}\right)^{2 n-3} p_{B}(1)+\left(1-2^{3-2 n} b^{2 n-3}\right) \cdot \frac{a+c}{2-b} \\
\left(\frac{b}{2}\right)^{2 n-2} p_{B}(1)+\left(1-2^{2-2 n} b^{2 n-2}\right) \cdot \frac{a+c}{2-b}
\end{array}\right]
\end{aligned}
$$

We take $p_{B}(1)=\frac{a+c}{2}+\frac{b}{2} p_{A}(1)$ into the above equation, and can obtain the dynamic adjustment model of dual-manufacturer price competition,

$$
\begin{aligned}
& p_{A}(n)=\left(\frac{b}{2}\right)^{2 n-3}\left[\frac{a+c}{2}+\frac{b}{2} p_{A}(1)\right]+\left(1-2^{3-2 n} b^{2 n-3}\right) \cdot \frac{a+c}{2-b} \\
& =2^{2-2 n} b^{2 n-2} p_{A}(1)+\left(1-2^{2-2 n} b^{2 n-2}\right) \cdot \frac{a+c}{2-b} \\
& p_{A}(n)=\left(\frac{b}{2}\right)^{2 n-3}\left[\frac{a+c}{2}+\frac{b}{2} p_{A}(1)\right]+\left(1-2^{3-2 n} b^{2 n-3}\right) \cdot \frac{a+c}{2-b} \\
& =2^{2-2 n} b^{2 n-2} p_{A}(1)+\left(1-2^{2-2 n} b^{2 n-2}\right) \cdot \frac{a+c}{2-b}
\end{aligned}
$$

If dual-oligopoly game times is infinite, i.e. $n \rightarrow \infty$, and the limitations of $p_{A}(n)$ and $p_{B}(n)$ confirmed in the above two equations exist, so the equilibrium solution exists, and the limitation is the equilibrium price.

$\lim _{n \rightarrow \infty} p_{A}(n)=\lim _{n \rightarrow \infty}\left[2^{2-2 n} b^{2 n-2} p_{A}(1)+\left(1-2^{2-2 n} b^{2 n-2}\right) \cdot \frac{a+c}{2-b}\right]$

$=\frac{a+c}{2-b}$ 
$\lim _{n \rightarrow \infty} p_{B}(n)=\lim _{n \rightarrow \infty}\left[2^{1-2 n} b^{2 n-1} p_{A}(1)+\left(1-2^{1-2 n} b^{2 n-1}\right) \cdot \frac{a+c}{2-b}\right]$

$=\frac{a+c}{2-b}$

It indicates that whatever the price that the first manufacturer enters into the market is, the equilibrium solution of the price model exists only, and the final adjusted price is the equilibrium price.

And we continue to analyze the dynamic change of dual-manufacturer price.

$$
\begin{aligned}
& p_{A}(n+1)=2^{-2 n} b^{2 n} p_{A}(1)+\left(1-2^{-2 n} b^{2 n}\right) \cdot \frac{a+c}{2-b} \\
& p_{B}(n+1)=2^{-1-2 n} b^{2 n+1} p_{A}(1)+\left(1-2^{-1-2 n} b^{2 n+1}\right) \cdot \frac{a+c}{2-b}
\end{aligned}
$$

Accordingly,

$$
\begin{aligned}
& p_{A}(n+1)-p_{A}(n)=2^{-2 n} b^{2 n-2}\left(b^{2}-4\right) p_{A}(1)+2^{-2 n} b^{2 n-3}(b+2)(a+c) \\
& =2^{-2 n} b^{2 n-2}\left(b^{2}-4\right)\left[p_{A}(1)-\frac{a+c}{2-b}\right] \\
& p_{B}(n+1)-p_{B}(n)=2^{-1-2 n} b^{2 n-1}\left(b^{2}-4\right)\left[p_{A}(1)-\frac{a+c}{2-b}\right]
\end{aligned}
$$

When $p_{A}(1)<\frac{a+c}{2-b}$, we can get from above two equations:

$$
\begin{aligned}
& p_{A}(n+1)-p_{A}(n)>0 \\
& p_{B}(n+1)-p_{B}(n)>0 .
\end{aligned}
$$

Therefore, when the price that the first manufacturer enters into the market is smaller than the equilibrium price, two manufacturers' prices strictly monotonically increase by degrees.

In the same way, when $p_{A}(1)>\frac{a+c}{2-b}$, we can get

$$
\begin{aligned}
& p_{A}(n+1)-p_{A}(n)<0 \\
& p_{B}(n+1)-p_{B}(n)<0 .
\end{aligned}
$$

i.e. when the price that the first manufacturer enters into the market is larger than the equilibrium price, two manufacturers' prices strictly monotonically decrease by degrees.

When $p_{A}(1)=\frac{a+c}{2-b}$, we can get

$$
\begin{aligned}
& p_{A}(n+1)=p_{A}(n)=\frac{a+c}{2-b} \\
& p_{B}(n+1)=p_{B}(n)=\frac{a+c}{2-b} .
\end{aligned}
$$

It indicates that the price that the first manufacturer enters into the market is the equilibrium price, two manufacturers' prices are changeless and kept at the level of the equilibrium price, in another words, and two manufacturers' prices have no dynamic changes, which achieve the equilibrium status at the very start.

\section{Conclusions}

Through the analysis of the equilibrium solution and price sequence dynamic changes of the Bertrand price competition model, we can get following conclusions.

(1) Under the condition of dual-manufacturer, however the first manufacturer initially selects the price, the equilibrium solution of the Bertrand model only exists.

(2) When the first manufacturer's initial price is $\frac{a+c}{2-b}$, two manufactures achieve the equilibrium at the very start. 
When the initial price deviates from the equilibrium price, the equilibrium process of dual-manufacture price is an infinite process, and the price adjustment sequence is the strictly monotonic sequence and the dual-manufacturer price adjustment changes at the same direction.

Otherwise, in this article, we give the dual-manufacturer price dynamic change model under the fixed demand function, and many problems such as manufacturer's profit change and price dynamic change under the condition of multi-manufacture need to be further studied.

\section{References}

Agliari A, Gardini L \& Puu T. (2000). The Dynamics of a Triopoly Cournot Game. Chaos, Solitons and Fractals. No.11(15). p.2531-2560.

Jean Tirole (French). (1997). The Theory of Industrial Organization. Beijing: China Renmin University Press.

Jianglin \& Liu, Zhaode. (2002). Pricing and Positioning Dynamic Game of Two Oligarchies. Forecasting. No.4. p.77-80.

Jiang, Qiyuan. (1993). Mathematical Model. Beijing: China Higher Education Press.

Peng, Yunfang. (2004). A Dynamic Game for Asymmetric Information based Oligarch Market Decision. China Soft Science. No.5. p.140-143.

Ren, Fangxu. (2005). A Study of Coordination and Competition in Bertrand Price Repeated Game. Journal of Zhongyuan Institute of Technology. No.1. p.33-36.

Tang, Xiaowo, Zengyong \& Li, Shiming. (2000). Management Economic Analysis: Theory and Application. Chengdu: University of Electronic Science and Technology of China Press.

Toshihiro Matsumura. (1999). Cournot Duopoly with Multiperiod Competition: Inventory as a Coordination Device. Australian Economic Papers. No.9. p.189-202.

Zhang, Yubo, Luo, Xianjue \& Xue, Junyi. (2003). Dynamic Cournot Model and Stability Analysis in Oligopoly Market. Systems Engineering-Theory \& Practice. No.11. p.55-60. 